\title{
Influence of service quality and involvement of a private indoor swimming pool on place attachment, place attitude, and relationship continuity intention
}

\author{
Kwang-Soo Lee ${ }^{1}$ \& Jung-Hee Jung ${ }^{2 *}$ \\ ${ }^{1}$ Soonchunhyang University \& ${ }^{2}$ Dankook University
}

\begin{abstract}
[Purpose] The purpose of this research is to empirically analyze the relationship between a indoor swimming pool on service quality and involvement, place attachment, place attitude \& relationship continuity intention through structural equation model analysis. [Methods] For this purpose, this study set 241 members at the five private indoor swimming pools located in Seoul as the research subjects. In an effort to verify the proposed structural model, this study used IBM SPSS WIN Ver. 21.0 and AMOS 18.0. [Results] As a result, First, the results showed that service quality had a positive effect on place identity. Second, it was found that service quality had a positive effect on place dependence. Third, the results showed that involvement didn't have a positive effect on place identity. Fourth, involvement didn't have a positive effect on place dependence. Fifth, the results showed that place identity had a positive effect on place attitude. Sixth, the results showed that place dependence had a positive effect on place attitude. Seventh, place attitude was found to had a positive effect on relationship continuity intention.
\end{abstract}

Key words: Service quality, Involvement, Place identity, Place dependence, Place attitude, Relationship continuity intention

\section{서 론}

최근 소비자들은 삶의 질을 향상시키려는 욕구가 증 가하면서 여가생활이나 자기개발을 위한 소비가 늘어나 고 있다. 특히 신체적·정신적·사회적 건강을 통한 개인 의 삶의 질 향상을 추구하기 위한 스포츠활동이 활발하 다(Ministry of Culture Sports and Tourism, 2017b). 2016년 국민생활체육 참여실태조사에 따르면 주 2회 이 상 규칙적 생활체육 참여율은 49.3\%로 2015년 대비 $4 \%$ 상승하였으며, 전 연령대에서 2015년 대비 참여율이 모두 상승하였다. 또한 체육활동 참여종목을 살펴보면,

논문 투고일 : 2018. 12. 23.

논문 수정일 : 2019. 01. 31.

게재 확정일 : 2019. 04. 03.

* 교신저자 : 정정희(jjhee4288@daum.net).

* 본 연구는 순천향대학교 학술연구비 지원으로 수행하였음.
걷기가 $35.6 \%$, 등산 $16.7 \%$, 보디빌딩 (헬스) $14.6 \%$, 축 구/풋살 $8.7 \%$, 수영 $7.4 \%$ 순으로 나타났고, 시간적 여 유가 된다면 참여하고 싶은 종목으로 수영이 $14.1 \%$ 로 가장 높은 선호도를 나타내고 있다(Ministry of Culture Sports and Tourism, 2017b). 수영장 사업체 수도 2015 년 279개에서 2016년 319개로 증가한 것으로 나타났 다(Ministry of Culture Sports and Tourism, 2017a). 이러한 소비자들의 수영에 대한 선호도와 시설의 증가는 관리자에게 서비스품질의 향상은 물론 소비자에게 수영 에 관한 관여도를 높여 실내수영장과 지속적인 관계를 유 지할 수 있도록 해야 한다. 이는 소비자들의 실내수영장 등의 스포츠시설에 관한 관심이 증대된 만큼 스포츠서비 스 분야에서 구매 후 행동에 영향을 미칠 수 있는 원인변 수로 소비자의 관여도가 매우 중요하다는 것을 강조한 연 구(Chung \& Choi, 2004)와 실내수영장 이용자의 서비 스품질이 구매행동의 하위변인으로 설정한 고객만족, 재 
방문의도 및 구전의도에 영향을 미친다는 연구(Seok, 2010)를 통해서 확인할 수 있다.

소비자들은 스포츠활동 장소와 지속적인 관계를 통해 서 연관성을 가지게 되며, 정서적 감정과 감정적 유대 감을 나타내는 장소애착을 형성하게 된다(Lee, 2011a). 장소애착은 장소 본질을 체험한 소비자의 감정, 인지, 경 험, 행동, 믿음의 상호작용으로 발생하는 감정적인 유대 관계이다(Kim, Song, \& Kim, 2015). 또한 조직 또는 개 인과 환경 사이의 긍정적인 결합을 의미하는 장소애착은 장소를 통해 소속감과 삶의 의미를 획득하며(Bricker \& Kerstetter, 2000), 특정한 행동을 추구하기 위해 특정 장 소나 환경에 장소애착을 느끼게 된다(Peterson, Stynes, Rosenthal, \& Dwyer, 1985). 이러한 장소애착은 두 가 지 요인으로 구성되어 있는데, 특정 장소를 방문한 소비 자의 감정이나 심리 또는 소속된 환경이나 문화적 상황 에 따라 장소애착 정도가 다르기 때문이다(Park, \& Kim, 2014). 첫 번째, 장소정체성 (place identity)은 개인의 목 적과 삶에 대한 의미 그리고 감정을 토대로 장소에 대한 개인의 감정적 몰입을 의미한다(Proshansky, Fabian, \& Kaminof, 1983). 두 번째, 장소의존성 (place dependence)은 개인이 특정 장소에 대해 느끼는 유대감의 정도 로 그 장소가 가진 특징과 함께 대체 가능한 다른 장소와 의 비교를 통해 나타나는 선호에 따라 결정된다(Williams, Roggenbuck, Patterson, \& Watson, 1992).

지속적인 스포츠활동을 하는 소비자들은 개개인이 선 호하거나 호감이 가는 장소에서 친근감을 표현하는 장소 애착을 보이게 된다(Kim, 2016). 장소애착은 개인을 둘 러싼 환경적 측면의 장소와 정서적 측면의 인간적 교감이 결합한 개념으로 장소 그 자체에서 저절로 형성되는 것이 아니라 개인의 인식, 경험, 심리적 반응, 상징행위 등에 의해서 형성된다(McAndrew, 1998). 이는 오랫동안 반복 적인 활동이나 시간을 전제로 형성되거나 짧은 경험이라 도 그 경험이 자신에게 의미를 지니게 된다면 장소애착 이 이루어진다(Manzo, 2005). 따라서 스포츠활동을 하 는 실내수영장에 대한 서비스품질을 긍정적으로 평가하 게 되면 장소애착에 긍정적인 영향을 미칠 것으로 기대할 수 있다. 즉 소비자들은 실내수영장에서 다양한 상호작용 과 이용 경험을 통해 형성된 서비스품질에 대한 평가가 긍정적이면 개인이 있고 싶어 하고 안전감과 편안함을 느끼는 특별한 장소에 대해 가지는 정서적인 유대인 장 소애착을 강하게 느끼게 될 것이다(Baek, 2016). 한편
소비자가 특정 대상에 대한 관련성이 높은 경우, 지속적 인 관여를 하게 되는데(Kim \& Park, 2015) 이는 소비 자가 스포츠활동을 하는 데 있어서 수영에 관여도가 높을 경우, 실내수영장에 대한 장소애착은 높아질 것으로 예 상할 수 있다. 이는 장소애착은 특정 장소를 상징하는 것 으로 중요하다고 생각하는 것이며, 물리적 공간에서 특정 한 활동이 이루어질 때 발생할 수 있기 때문이다. 따라서 이 연구에서는 이러한 장소와 관련된 애착을 장소정체성 과 장소의존성으로 구성하여(Williams \& Roggenbuck, 1989) 실내수영장의 서비스제공 정도를 측정하는 서비 스품질과 수영에 관한 지속적인 관심 정도인 관여도가 장 소애착에 긍정적인 영향을 미치는지 검증하고자 한다.

관광 및 여가-레크리에이션 분야에서 장소애착에 대한 연구는 관광객과 여가참여자의 목적지 선택과정에서 장 소애착 정도를 확인하거나, 장소애착이 여가활동을 촉진 하고 지속시키는 데 긍정적인 영향을 미친다고 보고하였 다(Alexandris, Kouthoris, \& Meligdis, 2006; Amendah \& Park, 2008; George \& George, 2004; Lee, 2011b; Park \& Kim, 2017; Park \& Choi, 2018). 또한 지각된 품 질과 장소정체성 및 장소의존성과의 긍정적 영향 관계를 밝힌 연구(Park \& Kim, 2014; Yoon, 2012; Lim, 2014) 와 관여도와 장소애착과의 정 $(+)$ 적인 관계를 검증한 연구 (Bricker \& Kerstetter, 2000; Gross \& Brown, 2008; Kyle, Bricker, Graefe, \& Wickham, 2004a)들을 찾아 볼 수 있다. 하지만 실내수영장 회원들을 대상으로 실내 수영장의 서비스품질과 관여도가 장소애착과의 관계를 통해 지속적인 관계유지를 위한 실무적 관점에서 마케 팅전략을 제시한 연구는 찾아보기 힘들어 이에 관한 연 구는 의미가 있을 것이다.

서비스품질은 서비스제공자의 인적업무 능력에 의존 하고 있고(Kandampulley, Mok, \& Sparks, 2001) 서비 스접촉과정에서 발생하는 고객과 서비스제공자의 상호 작용으로 결정되기 때문에 (Brown \& Swartz, 1989) 이 연구에서는 서비스품질은 실내수영장 강사 및 직원의 서 비스제공 정도를 의미한다. 또한 관여도는 소비자행동을 분석하는데 중요한 요인으로 특정 대상에 대한 개인의 중요성이나 관심 수준이다(Jung \& Kim, 2012). 과거의 경험이나 장기적 관심도에 의해 형성된 지속적 관여는 개인의 자아와 가치추구 성향과 관계가 있다(Dholakia, 2001). 이 연구에서는 관여도는 스포츠활동에 대한 지속 적인 흥미와 관심으로 형성된 애착을 의미하며, 지속적 
관여의 개념을 포함하고 있다. 따라서 소비자들은 실내수 영장의 서비스품질과 수영에 관한 관여도와 장소애착과 의 관계를 살펴보고자 한다.

장소애착은 장소와 이를 방문한 사람 간의 긍정적이 고 감정적인 유대관계로 해당 장소와의 친근성을 유지하 고 강한 상호관련성을 갖기 때문에 (Knez, 2005) 태도에 긍정적인 영향을 미칠 것이다. 특히 장소의존성은 개인의 욕구와 목표를 만족시키는 특정 장소의 잠재력과 관련된 기능적 애착으로 볼 수 있으며, 장소정체성은 장소에 대 한 강한 감정적 애착으로 특정 장소에 대해 의존성과 정 체성이 강할수록 장소에 대한 긍정적인 태도가 향상될 것 으로 예상할 수 있다(Park \& Kim, 2014; Baek, 2016). 또한 태도는 특정 대상에 대해 일관성 있는 호의적 또는 비호의적인 학습된 경향으로 이미 형성된 태도는 쉽게 변 화하지 않는다(Ajzen \& Fishbein, 2000). 따라서 장소에 대한 태도가 긍정적으로 형성되면 그 장소와의 관계를 지 속적으로 유지하려고 할 것으로 기대할 수 있다(Zhang, Inbakaran, Jackson, 2006; Son \& Jun, 2014). 이러한 선행연구결과들을 바탕으로 이 연구의 목적은 실내수영 장 고객이 지각하는 서비스품질과 관여도가 장소애착, 장 소태도 및 관계지속의도와 관계를 구조방정식모형을 통 해 실증적으로 검증하는 것이다. 이러한 연구결과는 수영 장경영에 있어 실내수영장 이용자 증대를 위한 실무에 필 요한 마케팅전략과 경쟁우위 확보에 필요한 시사점을 제 공할 수 있을 것이다.

\section{연구모형 및 가설}

\section{서비스품질과 장소애착과의 관계}

서비스품질은 소비자의 지각된 서비스와 기대 서비스 의 비교 및 평가로 소비자에 의해 지각되는 품질로 설명 하고 있다(Zeithaml, Berry, \& Parasuraman, 1996). 서 비스는 제품과 구별되는 고유의 특성으로 이질적이고 종 사원의 인적업무 능력에 의존하고 있기 때문에(Kwon, $\mathrm{Kim}, \&$ Yoon, 2012) 서비스접점에서 발생하는 고객과 종사원의 상호작용으로 결정된다고 할 수 있다(Brown $\&$ Swartz, 1989). 서비스품질과 관련된 선행연구들은 서비스를 제공하는 종사원의 태도나 전문능력 및 물리적 시설에 초점을 맞추고 있다(Parasuraman, Zeithmal, \& Berry, 1988). 따라서 소비자들은 종사원이 만족스러운 서비스를 제공하면 해당 장소와의 친근성을 유지하고 강
한 상호관련성을 갖게 된다(Knez, 2005). 즉 장소에 대 한 기억과 경험 속에 있는 심리적 접근을 통하여 얻는 감 정적인 유대관계인 장소애착을 갖게 된다(Kang \& Choi, 2012). 장소애착은 개인의 목적과 삶에 대한 의미 및 감 정을 토대로 장소에 대한 개인의 감정적 몰입을 의미하 는 장소정체성 (place identity)과 개인의 옥구를 만족시 키는 특정 장소와 관련된 기능적 애착을 의미하는 장소 의존성 (place dependence)으로 구분할 수 있다. 사람들 은 어떤 장소가 특정 활동을 하기에 좋은 곳이면 긍정적 인 평가를 한다(Williams et al., 1992). 따라서 실내수영 장에 대한 서비스품질 정도가 긍정적이면 물리적 공간에 대한 장소애착은 높아질 것이다.

서비스품질과 장소애착과의 관계를 실증적으로 검증 한 연구를 살펴보면, $\operatorname{Lim}(2014)$ 은 인천아시안게임의 브 랜드자산 요인인 지각된 품질이 장소정체성과 장소의존 성에 유의한 영향을 미친다고 보고하였다. Kim \& Kim (2014)은 박물관의 장소성 형성요인을 물리적 요인, 서 비스요인, 심리적 요인으로 구분하여 장소정체성과 장소 의존성과의 관계를 검증한 결과, 장소성 형성요인 모두 장소정체성에 영향을 미쳤으며, 물리적 요소가 가장 큰 영향을 미치는 것으로 나타났으나, 장소성 형성요인은 장 소의존성에 영향을 미치지 않는 것으로 나타났다. 또한 Park \& Kim(2014)은 향토음식 브랜드자산 요인 중 지 각된 품질이 장소정체성에 정 $(+)$ 의 영향을 미쳤지만, 지 각된 품질은 장소의존성에 정 $(+)$ 의 영향을 미치지 않았 다. $\operatorname{Kim}(2005)$ 은 문화관광축제 개최지의 서비스품질은 방문객의 장소애착심에 유의한 영향을 미친다고 하였다. 이러한 선행연구를 바탕으로 실내수영장에 대한 서비스 품질은 장소정체성과 장소의존성에 영향을 미칠 것으로 기대하며, 다음과 같은 가설을 설정하였다.

$\mathrm{H} 1$ : 서비스품질은 장소정체성에 긍정적인 영향을 미 칠 것이다.

$\mathrm{H} 2$ : 서비스품질은 장소의존성에 긍정적인 영향을 미 칠 것이다.

\section{관여도와 장소애착과의 관계}

관여도는 개인이 행위 및 활동에 관여하기 위해 가지는 개인적이고 주관적인 의미나 감성적인 애착으로(Schuett, 1993) 자아개념과 연관되어 있으며, 어떤 활동에 대한 특 별하고 구체적인 애착이나 태도를 설명하기도 한다. 따라 
서 관여의 정도가 높으면 자신의 정성을 특별한 일에 쏟 아 부을 가능성이 커진다(Son, 2016). 선행연구에서 관 여도가 높을수록 장소애착도가 향상된다고 보고하고 있 다(Bricker \& Kerstetter, 2000; Gross \& Brown, 2008; Kyle, Graefe, Manning, \& Bacon, 2004b). Kyle et al. (2004a)는 캠핑객들의 관여도가 장소애착과 정 $(+)$ 적인 관계를 갖는다고 하였고, Prayag \& Ryan(2012)은 모리 셔스섬을 방문한 관광객의 관여도가 장소애착에 유의한 영향을 미친다고 하였다. 또한 Choi, Jung, \& Han(2013) 의 연구에서는 관여도의 매력성, 자아표현, 삶의 중심성 요인이 장소정체성에 유의한 영향을 미친다고 보고하였 다. 이러한 연구결과는 수영에 대한 관여도가 높을수록 실내수영장에 대한 장소애착이 더 강해질 수 있을 것으로 기대할 수 있다. 따라서 다음과 같은 가설을 설정하였다.

$\mathrm{H} 3$ : 관여도는 장소정체성에 긍정적인 영향을 미칠 것 이다.

$\mathrm{H} 4$ : 관여도는 장소의존성에 긍정적인 영향을 미칠 것 이다.

\section{장소애착과 장소태도와의 관계}

장소애착은 장소와 방문한 사람 간의 긍정적이고 감 정적인 유대관계로 해당 장소와의 친근감을 유지하고 강 한 상호관련성을 갖게 한다(Knez, 2005). 장소애착은 방문객들의 편안함과 안전함을 고취하고 해당 장소에서 오랫동안 머물려 하는 성향을 높이기 때문에 (Hidalgo \& Hernandez, 2001). 장소에 대한 긍정적인 태도가 향상 될 것이다. Swann, Ronde, \& Hixon(1994)는 장소애착 은 특정 장소와 개인 간의 일치성을 높여 장소에 대해 긍 정적인 태도를 형성하고 기능적 애착을 경험함으로써 장 소에 대해 우호적 평가를 하게 된다고 하였다(Altman \& Low, 1992). Park \& Kim(2014)은 장소애착의 장소정 체성과 장소의존성 모두 지역에 대한 방문객 태도에 유 의한 정 $(+)$ 의 영향을 미친다고 보고하였다. Son \& Jun (2014)은 장소애착의 정소정체성은 장소브랜드태도에 유의한 영향을 미치는 것으로 나타났지만, 장소의존성은 장소브랜드태도에 유의한 영향을 미치지 않는다고 하였 다. 또한 Baek(2016)는 이벤트 방문객의 장소애착의 장 소정체성과 장소의존성이 개최지태도에 유의미한 영향 을 미친다고 보고하였다. 이러한 선행연구를 바탕으로 실 내수영장에 대한 장소애착 요인인 장소정체성과 장소의
존성이 높을수록 실내수영장에 대한 태도가 긍정적일 것 으로 기대할 수 있을 것이다. 따라서 다음과 같은 가설을 설정하였다.

$\mathrm{H} 5$ : 장소정체성은 장소태도에 긍정적인 영향을 미칠 것이다.

$\mathrm{H} 6$ : 장소의존성은 장소태도에 긍정적인 영향을 미칠 것이다.

\section{장소태도와 관계지속의도와의 관계}

태도는 개인의 행동을 이해하기 위해 일관된 규칙성 을 관찰하여 미래 행동을 예측하거나 설명할 때 유용한 요인이다(Britannica, 1977). 태도를 특정 장소에 대한 개인의 주관적인 관점에서 살펴보면, 특정 장소와 관련하 여 호의적인 태도가 형성되면 긍정적인 행동이 증가할 것 이다. 특정 장소태도와 관계지속의도와의 관계를 직접적 으로 검증한 연구는 찾아보기 힘들지만, 다음과 같은 선 행연구로 유추할 수 있을 것이다. Zhang et al.(2006)은 목적에 대한 태도는 우호적인 방문태도와 구매의도의 증 가로 나타난다고 하였다. Son \& Jun(2014)은 장소브랜 드태도는 방문의도에 유의한 영향을 미친다고 보고하였 고, Choi \& Choi(2018)는 지역축제 개최지에 대한 태도 는 개최지충성도에 유의한 영향을 미친다고 하였다. Lee (2012)은 골프용품 매장태도가 충성도에 긍정적인 영향 을 미친다고 하였다. 이러한 선행연구를 바탕으로 이 연 구에서는 실내수영장에 대한 장소태도가 긍정적일수록 관계를 지속하고자 하는 의도는 향상될 것으로 기대할 수 있다. 따라서 다음과 같은 가설을 설정하였다.

$\mathrm{H} 7$ : 장소태도는 관계지속의도에 긍정적인 영향을 미 칠 것이다.

이상의 내용을 그림으로 도식화하면 〈Fig. 1)과 같다.

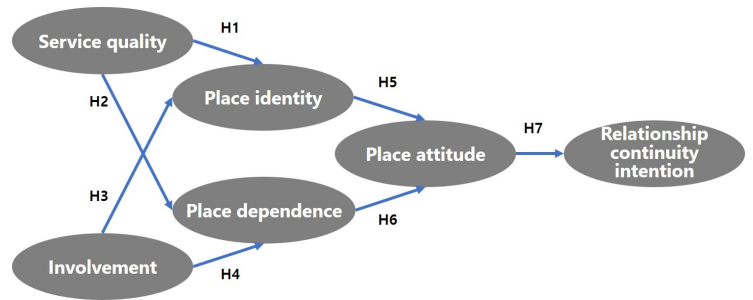

Fig. 1. The hypothesized model 


\section{연구방법}

\section{표본 및 자료수집}

이 연구에서는 구·시에서 운영하는 공공 실내수영장 이 아닌 개인이 운영하는 사설 실내수영장으로 연구대상 을 설정하였다. 또한 대규모 복합 스포츠시설 내 부속 실 내수영장을 제외한 단일 사설 실내수영장의 남녀 회원 (이용기간 3개월 이상)을 설문조사 대상으로 하였다. 자 료수집은 서울에 있는 사설 실내수영장 5 개소에서 각각 50 명씩 총 250 명을 대상으로 하였다. 설문조사는 사전 에 교육을 받은 조사원 2명이 사설 실내수영장에 직접 방 문하여 회원들에게 설문조사의 목적과 내용을 설명한 후 실시하였다. 표본추출은 편의표본추출법과 설문지작성 은 자기기입식으로 하였다. 조사 기간은 2018년 5월 1일 $\sim 6$ 월 30 일까지 실시하였으며, 총 250 부의 설문지 중 불 성실한 9 부를 제외한 241 부가 유효하여 최종통계분석 에 이용되었다. 조사대상의 일반적 특성에 관한 내용은 〈Table 1〉과 같다.

Table 1. Characteristics of respondents

\begin{tabular}{|c|c|c|c|}
\hline Attributes & Content & Number & Frequency $(\%)$ \\
\hline \multirow{2}{*}{ Gender } & Male & 105 & 43.6 \\
\hline & Female & 136 & 56.4 \\
\hline \multirow{5}{*}{ Age } & Twenties & 14 & 5.8 \\
\hline & Thirties & 98 & 40.7 \\
\hline & Forties & 53 & 22.0 \\
\hline & Fifties & 54 & 22.4 \\
\hline & Over Sixties & 22 & 9.1 \\
\hline Average of Age & & 43.8 & \\
\hline \multirow{6}{*}{$\begin{array}{l}\text { Swimming Pool } \\
\text { Use Time }\end{array}$} & Below 1 year & 105 & 43.5 \\
\hline & Below 1 3 years & 64 & 26.6 \\
\hline & Below 3 5 years & 43 & 17.8 \\
\hline & Below $5 \sim 7$ years & 7 & 2.9 \\
\hline & Below 7 10 years & 17 & 7.1 \\
\hline & Over 10 years & 5 & 2.1 \\
\hline \multirow{6}{*}{ Occupation } & Employed & 96 & 39.8 \\
\hline & Own business & 43 & 17.8 \\
\hline & Not employed & 36 & 14.9 \\
\hline & House worker & 33 & 13.7 \\
\hline & Others & 25 & 10.4 \\
\hline & university student & 8 & 3.3 \\
\hline
\end{tabular}

\section{구성개념의 조작적 정의 및 측정}

이 연구에서 사용한 변수들은 관련 분야의 연구에서 이미 신뢰도와 타당도가 검증된 측정도구를 사용하였으 며, 이 연구의 목적에 맞게 수정하여 사용하였다. 자세한 설문문항은 〈Table 2〉에 제시하였다.

서비스품질 (service quality)은 실내수영장의 서비스 제공자(강사 및 직원 포함)의 서비스제공 정도라 정의하 였다. 이를 측정하기 위해 Parasuraman et al.(1988), $\operatorname{Sbpetim(2012)ㅇㅢ~ㅇㅕㄴㄱㅜㄹㅡㄹ~ㅂㅏㅌㅏㅇㅇㅡㄹㅗ~} \operatorname{Kim} \& \operatorname{Jung}(2017)$ 가 사용한 측정 문항을 이 연구의 목적에 맞게 수정하 여 5 문항으로 구성하였다. 관여도(involvement)는 현재 스포츠활동을 하는 수영에 관해 형성되는 지속적인 관심 정도라 정의하였다. 이를 측정하기 위해 Zaichkowsky (1985)가 개발한 척도를 바탕으로 Lee \& Jung(2015) 의 연구에서 사용한 측정항목을 이 연구의 목적에 맞게 수 정하여 5 문항으로 구성하였다. 장소애착 (place attachment)은 실내수영장에서 경험하는 다양한 형태의 태도 나 감정 또는 결과를 통해 장소에 대한 장소정체성과 장 소의존성으로 구분하였다. 장소정체성은 현재 이용하고 있는 실내수영장에 대하여 회원이 인식하는 감정적인 애 착 정도라 정의하였다. 장소의존성은 현재 이용하고 있 는 실내수영장에 대해 느끼는 기능적 애착 정도라 정의하 였다. 이를 측정하기 위해서 Brown \& Raymond(2007), Gross \& Brown(2008), Baek(2016)의 연구에서 사용한 측정항목을 이 연구의 목적에 맞게 수정하여 각각 5 문항 씩 10문항으로 구성하였다. 장소태도(place attitude)는 실내수영장에 대한 긍정적 혹은 부정적 반응으로 정의하 였다. 이를 측정하기 위해 Pan \& Schmitt(1996)의 연 구를 바탕으로 Lee \& Jung(2015)가 사용한 측정항목 을 이 연구의 목적에 맞게 수정하여 4 문항으로 구성하였 다. 관계지속의도(relationship continuity intention)는 현재 이용 중인 실내수영장과 우호적인 관계를 맺으며 관 계를 유지하려는 정도라 정의하였다. 이를 측정하기 위해 Kim \& Jung(2018)와 Kim \& Kwon(2011)의 연구에서 사용한 측정항목을 이용하여 4 문항으로 구성하였다. 또 한 조사대상의 일반적인 특성을 살펴보기 위해서 성별, 연령, 직업, 실내수영장 이용기간 등 4 문항을 포함시켰다. 이 연구의 구성 개념들은 1 (전혀 아니다)에서 5(매우 그 렇다)까지의 범위를 갖는 리커트식 5 점 척도와 장소태도 는 의미분별척도로 측정하였다. 
Table 2. Confirmatory factor analysis and reliabilities

\begin{tabular}{|c|c|c|c|c|c|c|c|}
\hline Factors & Items & $\mathrm{SC}$ & SE & $t$-value & $\alpha$ & $\mathrm{CR}$ & AVE \\
\hline \multirow{5}{*}{$\begin{array}{l}\text { Service } \\
\text { quality }\end{array}$} & Swimming pool readily grands requirements from the members." & - & - & - & \multirow{5}{*}{.919} & \multirow{5}{*}{.931} & \multirow{5}{*}{.772} \\
\hline & Swimming pool rapidly responds to requests by the members. & .876 & .191 & - & & & \\
\hline & Swimming pool provides high-quality services to the members. & .892 & .172 & 19.149 & & & \\
\hline & $\begin{array}{l}\text { Swimming pool provides individual services to satisfy the desires of the } \\
\text { members. }\end{array}$ & .871 & .211 & 18.310 & & & \\
\hline & Swimming pool provides rapid service to the members. & .806 & .303 & 15.929 & & & \\
\hline \multirow{5}{*}{ Involvement } & Swimming is important to me.* & - & - & - & \multirow{5}{*}{.901} & \multirow{5}{*}{.840} & \multirow{5}{*}{.570} \\
\hline & Swimming is related to me. & .710 & .812 & - & & & \\
\hline & Swimming is worth to me. & .883 & .392 & 12.968 & & & \\
\hline & Swimming is useful to me. & .893 & .401 & 13.103 & & & \\
\hline & I am interested in swimming. & .853 & .508 & 12.574 & & & \\
\hline \multirow{5}{*}{$\begin{array}{l}\text { Place } \\
\text { identity }\end{array}$} & Swimming pool is part of me. & 619 & .532 & - & \multirow{5}{*}{.837} & \multirow{5}{*}{.875} & \multirow{5}{*}{.643} \\
\hline & Swimming pool is a meaningful place for me. & - & - & - & & & \\
\hline & Swimming pool is a special place for me. & 632 & .491 & 8.430 & & & \\
\hline & Swimming pool tells me who I am. & .857 & .250 & 10.555 & & & \\
\hline & I feel attached to the swimming pool. & .947 & .065 & 10.938 & & & \\
\hline \multirow{5}{*}{$\begin{array}{l}\text { Place } \\
\text { dependence }\end{array}$} & Swimming pool currently using is better than any other pool. & - & - & - & \multirow{5}{*}{.858} & \multirow{5}{*}{.885} & \multirow{5}{*}{.664} \\
\hline & $\begin{array}{l}\text { Swimming pool currently using is more satisfactory than other swimming } \\
\text { pools. }\end{array}$ & .784 & .369 & - & & & \\
\hline & Experience in the current pool can not be replaced by another pool. & .912 & .133 & 15.030 & & & \\
\hline & $\begin{array}{l}\text { Swimming pool currently using is good for environment for leisure } \\
\text { activities. }\end{array}$ & .830 & .254 & 13.821 & & & \\
\hline & Swimming pool currently using is the best place for leisure activities. & .588 & .500 & 9.207 & & & \\
\hline \multirow{4}{*}{$\begin{array}{l}\text { Place } \\
\text { attitude }\end{array}$} & I think the swimming pool positively/I think the swimming pool negatively & .909 & .169 & - & \multirow{4}{*}{.947} & \multirow{4}{*}{.945} & \multirow{4}{*}{.811} \\
\hline & Like/Dislike & .957 & .081 & 26.092 & & & \\
\hline & Good/Bad & .902 & .194 & 22.477 & & & \\
\hline & Favorabole/unfavorable & .823 & .311 & 9.207 & & & \\
\hline \multirow{4}{*}{$\begin{array}{l}\text { Relationship } \\
\text { continuity } \\
\text { intention }\end{array}$} & I will continue to maintain friendly relations with a swimming pool. & .901 & .137 & - & \multirow{4}{*}{.907} & \multirow{4}{*}{.921} & \multirow{4}{*}{.747} \\
\hline & I will maintain continued relations with a swimming pool. & .894 & .151 & 19.497 & & & \\
\hline & I won't readily change a swimming pool. & .821 & .249 & 16.747 & & & \\
\hline & I will continuously take exercise at a swimming pool. & .710 & .408 & 13.039 & & & \\
\hline
\end{tabular}

means item deleted in confirmatory factor analysis

\section{자료분석}

이 연구에서는 조사대상의 특성을 파악하기 위해 빈 도분석, Cronbach's a검사를 위해 IBM SPSSWIN Ver. 21.0 과 판별타당성을 검증하기 위해 상관관계분석, 확 인적요인분석과 구조방정식모형분석을 위해 $\mathrm{AMOS} 18.0$ 을 활용하였다.

\section{연구결과}

\section{구성개념의 신뢰성 및 타당성 분석}

이 연구에서는 다 항목을 이용한 각 측정항목에 대한 단일차원성을 검증하기 위해 확인적요인분석을 실시하 였다. 즉 수정지수 $(\mathrm{MI})$ 를 이용하여 최적모형을 도출하기 
위한 척도정제과정을 진행하였다. 수정지수 값 ' 10 '을 기 준으로 척도정제를 실시하였고, 측정항목 오차항의 공분 산관계에서도 공분산 수정지수가 '10'이상을 기준으로 척도를 정제하였다. 먼저 수정지수의 누적합계를 최대가 되는 오차항과 그에 해당하는 변수를 제거해 가며 적합 도를 개선하였다. 그 결과 서비스품질 1 , 관여도 1 , 장소 정체성 2 , 장소의존성 1 의 4 항목을 제거하였다. 척도정 제과정을 통해 도출된 확인적요인분석 결과, $\chi^{2} / d f=$ $413.380 / 234(p=.000), \mathrm{GFI}=.875, \mathrm{CFI}=.960$, $\mathrm{TLI}=.953, \mathrm{RMR}=.074, \mathrm{RMSEA}=.057$ 로 일반적인 추천기준치 $(\mathrm{GFI} \geq .90 ; \mathrm{CFI} \geq .90 ; \mathrm{TLI} \geq .90 ; \mathrm{RMR}$, $\mathrm{RMSEA} \leq .05 ; \chi^{2}$ 작을수록 바람직, $p$ 값 $\left.\geq .05\right)$ 를 충족하 거나 가깝게 나타나 대체적으로 우수한 적으로 나타났다 (Bagozzi \& Yi, 1988). 또한 도출된 측정항목의 신뢰도 분석결과, Cronbach's a값이 .837 .947로 .7이상으로 나타나 내적일관성이 있는 것으로 확인되었다(Nunnally $\&$ Bernstein, 1994). 각 구성개념의 수렴타당성을 확인 하기 위해 평균분산추출값 $(\mathrm{AVE})$ 과 개념신뢰도 $(\mathrm{CR})$ 를 산출한 결과, 모든 변수가 일반적인 기준 $(\mathrm{AVE}=.5$ 이상, $\mathrm{CR}=.7$ 이상 $)$ 을 충족시켰다. 따라서 각각의 구성개념에 대한 항목들은 수렴타당성이 확보되었다고 할 수 있다. 자세한 내용은 〈Table 2〉와 같다.

상관관계분석 결과, 모든 변수가 정 $(+)$ 의 상관관계 ( $p<.01)$ 를 가지는 것으로 확인되었다. 또한 $\mathrm{AVE}$ 값이 상관계수 제곱값을 초과하면 판별타당성이 확보된다 (Fornell \& Larcker, 1981). 그 결과, AVE값은 .570 .811 으로 상관계수 제곱값인 .056 .401(.236 $\left.~ .633^{2}\right)$ 을 모두 초과하여 판별타당성이 확보되었다. 자세한 내용 은 〈Table 3〉과 같다.

\section{가설 검증 및 논의}

가설검증을 위해 구조방정식모형을 설정하고 구성개 념 사이의 관계에 대한 경로분석을 하였다. 연구모형의 적합도 수치는 $\chi^{2} / d f=313.077 / 196,(p=.000), \mathrm{GFI}=$ $.897, \mathrm{CFI}=.972, \mathrm{TLI}=.967 \mathrm{RMR}=.112, \mathrm{RMSEA}=$ .050으로 확인되었다. 특히 표본의 수에 영향을 받지 않 는 지표인 $\mathrm{CFI}$ 와 TLI의 결과가 이상적이기 때문에 구성 개념 사이의 영향 관계를 설명하기에 적절한 것으로 판단 된다(Lee \& Hwang, 2017).

가설 1 의 '서비스품질은 장소정체성에 긍정적인 영향 을 미칠 것이다'는 경로계수 $=.554$, 값 $=6.516$ 으로 긍 정적인 영향을 미치는 것을 확인되어 채택되었다. 이러한 결과는 실내수영장 서비스제공에 대한 회원의 요구와 옥 구충족 정도가 긍정적일수록 실내수영장에 대하여 회원 이 인식하는 감정적인 애착 정도가 높아진다는 것이다. 선행연구에서 실내수영장 서비스품질과 장소정체성과의 관계를 실증적으로 검증한 연구는 찾아보기 힘들지만, 관 광지나 여가시설에 관한 선행연구를 통해서 관계를 검토 하고자 한다. $\operatorname{Kim} \& \operatorname{Kim}(2014)$ 은 박물관의 장소성 형 성요인을 물리적 요인, 서비스요인, 심리적 요인으로 구 분하여 장소정체성과 장소의존성과의 관계를 검증한 결 과, 장소성 형성요인 모두 장소정체성에 영향을 미쳤으 며, 물리적 요소가 가장 큰 영향을 미치는 것으로 나타났 다. 또한 Park \& Kim(2014)은 향토음식 브랜드자산 요 인 중 지각된 품질이 장소정체성에 정 $(+)$ 의 영향을 미친 다는 것과 같은 결과이다. 따라서 실내수영장을 이용하는 회원들의 감정적인 애착을 높이기 위해서는 회원들이 안 전하게 수영을 즐기고 원하는 서비스를 신속하게 제공하

Table 3. Correlations among variables

\begin{tabular}{|c|c|c|c|c|c|c|}
\hline Factors & $\begin{array}{l}\text { Service } \\
\text { quality }\end{array}$ & Involvement & $\begin{array}{l}\text { Place } \\
\text { identity }\end{array}$ & $\begin{array}{c}\text { Place } \\
\text { dependence }\end{array}$ & $\begin{array}{l}\text { Place } \\
\text { attitude }\end{array}$ & $\begin{array}{l}\text { Relationship } \\
\text { continuity } \\
\text { intention }\end{array}$ \\
\hline Service quality & 1 & & & & & \\
\hline Involvement & $.482^{* *}$ & 1 & & & & \\
\hline Place identity & $.551^{* *}$ & $.287^{* *}$ & 1 & & & \\
\hline Place dependence & $.509^{* * *}$ & $.236^{* *}$ & $.489^{* *}$ & 1 & & \\
\hline Place attitude & $.486^{* *}$ & $.336^{* *}$ & $.343^{* *}$ & $.397^{* *}$ & 1 & \\
\hline Relationship continuity intention & $.520^{* *}$ & $.370^{* *}$ & $.347^{* *}$ & $.277^{* *}$ & $.633^{* *}$ & 1 \\
\hline
\end{tabular}


여 종사원들의 서비스 질을 향상시켜야 한다.

가설 2 의 '서비스품질은 장소의존성에 긍정적인 영향 을 미칠 것이다'는 경로계수 $=.529, t$ 값 $=6.777$ 로 긍 정적인 영향을 미치는 것으로 확인되어 채택되었다. 이러 한 결과는 실내수영장 서비스제 공에 대한 긍정적인 평가 가 높을수록 회원들은 실내수영장에 대해 시설과 환경 및 여가활동에 적합한 장소로써 의존성이 높아진다는 것 이다. Yoon(2011)는 브랜드자산 요인 중 지각된 품질이 장소애착의 장소의존성에 유의한 영향을 미친다고 하였 다. 또한 $\operatorname{Lim}(2014)$ 은 인천아시안게임의 브랜드자산 요 인인 지각된 품질이 장소의존성에 유의한 영향을 미친다 고 보고하여 이 연구를 뒷받침해 준다. 따라서 실내수영 장 관리자는 회원들이 여가활동 장소로써 의존성을 높이 기 위해서는 시설의 편리함과 신속한 서비스 등 양질의 서비스를 제공하여야 할 것이다.

가설3의 '관여도는 장소정체성에 긍정적인 영향을 미 칠 것이다'는 경로계수 $=-.021, t$ 값 $=-.298$ 로 긍정적 인 영향을 미치지 않는 것으로 확인되어 기각되었다. 이러 한 결과는 회원들이 수영에 관한 관심 정도가 높다고 해 도 실내수영장을 특별한 장소로써 의미있게 느끼지 않는 다는 것이다. 또한 가설 4 의 '관여도는 장소의존성에 긍 정적인 영향을 미칠 것이다'는 경로계수 $=.-022, t$ 값 $=$ -.301로 긍정적인 영향을 미치지 않는 것으로 확인되어 기각되었다. 이는 회원들은 수영을 가치 있고 중요하게 인 식하며, 본인에게 유용하고 관련이 높다고 해도 실내수 영장의 시설과 환경, 여가활동에 적합한 장소로써 의존 성이 높지 않다는 것이다.

이는 관여도가 장소애착에 긍정적인 영향을 미친다는
선행연구와 다른 결과이다(Bricker \& Kerstetter, 2000; Choi et al., 2013; Gross \& Brown, 2008; Kim, 2017; Kyle et al., 2004b). 이러한 결과의 배경으로는 사설 실내 수영장이 갖는 스포츠활동 공간으로서의 특성을 살펴볼 필요가 있다. 사설 실내수영장은 회원들의 건강과 여가 생활을 위한 장소로 거주지에서 근접하고 회원등록을 한 후에 특별한 비용이 거의 없으며, 타인의 강요가 아닌 자 신의 옥구나 필요로 참여해서 내재적인 보상과 단기적인 쾌락을 얻을 수 있으므로 수영 자체에 대한 중요성이나 관심 수준은 높지만, 실내수영장에 대해 느끼고 있는 장 소애착은 높지 않다는 것이다. 이와 관련해서 활동여가 에 관한 선행연구에서 일상적 여가보다 좀 더 전문성과 적극적인 여가활동인 캠핑, 익스트림 스포츠 등 진지한 여가에서 여가활동 대상지에 대해 긍정적인 경우가 많았 다(Lee, 2011b). 따라서 스포츠활동 강도와 전문성에 따 라 관여도가 장소애착에 미치는 영향이 다를 수 있음을 시사하고 있다.

가설 5 의 '장소정체성은 장소태도에 긍정적인 영향을 미칠 것이다'는 경로계수 $=.193, t$ 값 $=2.576$ 으로 긍정 적인 영향을 미치는 것으로 확인되어 채택되었다. 이러한 결과는 실내수영장에 대한 회원들의 감정적인 애착이 긍 정적이면 장소태도가 긍정적으로 반응한다는 것이다. 이 는 Park \& Kim(2014)의 장소정체성이 지역에 대한 방 문객 태도에 유의한 정 $(+)$ 의 영향을 미친다는 것과 Son $\& \operatorname{Jun}(2014)$ 의 연구에서 정소정체성이 장소브랜드태 도에 유의한 영향을 미치는 결과와 같은 맥락의 결과이 다. 따라서 관리자는 실내수영장과 회원 간의 긍정적이 고 감정적인 유대관계가 형성될 수 있도록 강사와 종사

Table 4. Testing of the proposed hypotheses

\begin{tabular}{|c|c|c|c|c|c|c|}
\hline Hypotheses & Paths & $\mathrm{SC}$ & SE & $t$ & $p$ & Acceptance \\
\hline $\mathrm{H} 1$ & Service quality $\rightarrow$ Place identity & .554 & .063 & 6.516 & .000 & Accepted \\
\hline $\mathrm{H} 2$ & Service quality $\rightarrow$ Place dependence & .529 & .057 & 6.777 & .000 & Accepted \\
\hline $\mathrm{H} 3$ & Involvement $\rightarrow$ Place identity & -.021 & .034 & -.298 & .766 & Rejected \\
\hline $\mathrm{H} 4$ & Involvement $\rightarrow$ Place dependence & -.022 & .046 & -.301 & .763 & Rejected \\
\hline H5 & Place identity $\rightarrow$ Place attitude & .193 & .118 & 2.576 & .010 & Accepted \\
\hline H6 & Place dependence $\rightarrow$ Place attitude & .316 & .089 & 4.156 & .000 & Accepted \\
\hline $\mathrm{H7}$ & Place attitude $\rightarrow$ Relationship continuity intention & .624 & .053 & 10.187 & .000 & Accepted \\
\hline \multicolumn{7}{|c|}{ Fit: $\chi^{2}=313.077, d f=196, p=.000, \mathrm{GFI}=.897, \mathrm{CFI}=.972, \mathrm{TLI}=.967 \mathrm{RMR}=.112, \mathrm{RMSEA}=.050$} \\
\hline
\end{tabular}


원들은 회원과의 친근성을 유지하고 강한 상호관련성을 갖도록 노력해야 한다.

가설 6 의 '장소의존성은 장소태도에 긍정적인 영향을 미칠 것이다'는 경로계수 $=.316, t$ 값 $=4.156$ 으로 긍정 적인 영향을 미치는 것으로 확인되어 채택되었다. 이러한 결과는 현재 이용하고 있는 실내수영장에 대해 느끼는 기 능적 애착이 높으면 수영장에 대한 긍정적인 태도가 높아 진다는 것이다. 이는 $\operatorname{Baek}(2016)$ 의 이벤트 방문객의 장 소의존성이 개최지태도에 유의미한 영향을 미친다고 보 고한 것과 Park \& Kim(2014)의 장소의존성이 지역에 대한 방문객 태도에 유의한 정 $(+)$ 의 영향을 미친다는 것 과 같은 의미의 결과이다. 따라서 관리자는 회원들이 실 내수영장을 이용할 때 다른 수영장보다 편안하고 안전하 게 여가활동을 할 수 있는 환경을 만들어야 긍정적인 태 도가 형성될 것이다.

가설7의 '장소태도는 관계지속의도에 긍정적인 영향 을 미칠 것이다'는 경로계수 $=.624, t$ 값 $=10.187$ 로 긍 정적인 영향을 미치는 것으로 확인되어 채택되었다. 이러 한 결과는 실내수영장에 대한 긍정적인 태도가 향상되면 수영장과 관계를 지속하고자 하는 의도가 향상된다는 것 이다. 이는 Gilboa \& Herstein(2012)의 연구에서 장소에 대해 우호적 평가를 하면 장소에 대한 방문 및 충성도로 이어진다는 연구결과와 Lee(2012)의 골프용품 매장태 도가 충성도에 긍정적인 영향을 미친다는 결과, Choi \& Choi (2018)의 지역축제 개최지에 대한 태도가 개최지충 성도에 유의한 영향을 미친다는 것과 같은 맥락의 결과 이다. 따라서 실내수영장을 긍정적인 장소로 평가할 수 있도록 관리자는 회원들이 수영을 통해서 즐거움과 행복 감 등의 긍정적인 경험을 할 수 있도록 물리적·쾌락적 조 건을 갖추도록 노력해야 한다.

\section{결 론}

\section{연구의 시사점}

이 연구의 결과는 다음과 같은 시사점을 갖는다. 첫째, 서비스품질은 장소정체성과 장소의존성에 긍정적인 영 향을 미치는 것으로 나타났다. 이는 실내수영장 서비스 품질에 대해 긍정적으로 평가하면 회원들은 실내수영장 에 장소애착이 형성된다는 것을 시사한다. 둘째, 관여도
는 장소정체성과 장소의존성에 긍정적인 영향을 미치지 않는 것으로 나타났다. 이는 회원들에게 실내수영장에 관 한 관심과 흥미를 높이는 방안을 고려하여 물리적, 쾌락 적 여가활동 공간으로써 장소애착을 강화하여야 한다는 것을 시사한다. 셋째, 장소정체성과 장소의존성은 장소 태도에 긍정적인 영향을 미치는 것으로 나타났다. 이는 실내수영장에서 느낄 수 있는 특별한 장소로써 안전감과 만족감 등의 장소애착이 실내수영장에 대한 우호적인 장 소태도를 형성하게 된다는 것을 시사한다. 넷째, 장소태 도는 관계지속의도에 긍정적인 영향을 미치는 것으로 나 타났다. 실내수영장에 대한 우호적 평가는 장기적인 관계 로 발전하게 된다는 것을 시사한다. 이 연구에서는 실내 수영장에 대한 장소적 측면에서 장소애착을 강화하기 위 해서 회원이 지각하는 수영에 관한 관여도보다 서비스품 질 향상에 노력해야 한다는 것을 확인하였다. 또한 장소 애착이 장소태도에 영향을 미치고 장소태도가 관계지속 의도에 영향을 미치는 중요한 변인이라는 것을 확인하였 고, 이는 공간적인 특수성을 반영한 다양한 추후 연구가 필요하다는 것을 시사한다.

\section{연구의 한계점}

이 연구에서는 몇 가지 한계점을 가지고 있다. 첫째, 수도권에 있는 5 개소의 상업 실내수영장 회원 241명을 대상으로 연구를 진행하였기 때문에 연구결과를 일반화 하기에 무리가 있다. 후속연구에서는 지역과 대상을 확대 하여 외적타당성을 높여야 한다. 둘째, 장소애착에 영향 을 미칠 수 있는 동기나 장소성 형성요인 등 다양한 변수 를 고려하여 분석한다면 의미 있는 결과를 도출할 수 있 을 것이다. 셋째, 장소애착에 대한 연구는 선행연구가 양 적인 측면에서 부족하며, 특히 스포츠시설과 관련된 연구 는 찾아보기 힘들다. 따라서 타 학문 분야의 선행연구에 서 제시된 다양한 논의를 실내수영장의 장소적 의미를 구 체적으로 밝히는 데 한계가 있다. 다양한 연구모형으로 장소로서의 스포츠시설에 관한 후속 연구가 계속해서 이 루어져야 할 것이다.

\section{참고문헌}

Ahn, D. H. (2001). Service marketing. Seoul: Dea Nam. Ajzen, I., \& Fishbein, M. (2000). Attitudes and the attitude- 
behavior relation: Reasoned and automatic process. European Review of Social Psychology, 11(1), 1-33.

Alexandris, K., Kouthoris, C., \& Meligdis, A. (2006). Increasing customers' loyalty in a skiing resort. International Contemporary Hospitality Management, 18(5), 414-425.

Altman, I., \& Low, S. M. (1992). Place attachment: Human behavior and environment. NY: Plenum Press.

Amendah, E., \& Park, J. (2008). Consumer involvement and psychological antecedents on eco-friendly destinations: Willingness to pay more. Journal of Hospitality \& Leisure Marketing, 17(3-4), 262-283.

Baek, K. M. (2016). The influence of event visitor's experiential value on emotional response, place attachment and attitude toward host region: Focused on the visitors of SUNCHEON BAY NATIONAL GARDEN EXPO. Event \& Convention Research, 12(2), 1-24.

Bricker, K., \& Kerstetter, D. (2000). Level of specialization and place attachment: An exploratory study of whitewater recreationists. Leisure Sciences, 22, 233-257.

Britannica, E. (1977). The new encyclopaedia britannica. Encyclopaedia Britannica Inc.

Brown, G., \& Raymond, C. (2007). The relationship between place attachment and landscape values: Toward mapping place attachment. Applied Geography, 27, 89-111.

Brown, S. W., \& Swartz, T. A. (1989). A gap analysis of hotel and restaurant administration quality. Journal of Marketing, 53(2), 92-98.

Choi, K. J., \& Choi, H. G. (2018). The effects of brand knowledge of regional festival on the image, attitude and loyalty of region: Focusing on the Boryeong mud festival. Korean Review of Corporation Management, 9(1), 207-223.

Choi, S. B., Jung, W. J., \& Han, T. Y. (2013). The relationships of involvement and destination's place attachment and loyalty of Gangwon province east sea beach visitor. Korean Journal of Sports Science, 22(5), 197-210.

Choi, Y. H., Lee, W. C., \& Lee, H. (2005). Community attachment and attitudes of residents on public show-caves. Korean Journal of Hotel Administration, 14(1), 193-215.

Chung, E. K., \& Choi, Y. J. (2004). The relationship between consumers' participation, services satisfaction and clients' loyalty of indoor swimming pools: Focused on the control effects of participation. Korean Education Inquiry, 19, 81-100.

Dholakia, U. M. (2001). A motivational process model of product involvement and consumer risk perception. European Journal of Marketing, 35(11/12), 1340-1360.
Fornell, C., \& Larcker, D. F. (1981). Evaluating structural equation models with unobservable variables and measurement error. Journal of Marketing Research, 18(1), 39-50.

George, B. P., \& George, B. P. (2004). Past visit and the intention to revisit a destination: Place attachment as the mediator and novelty seeking as the moderator. The Journal of Tourism Studies, 15(2), 51-66.

Gilboa, S., \& Herstein, R. (2012). Place status, place loyalty and well being: An exploratory investigation of Israeli residents. Journal of Place Management and Development, 5(2), 141157.

Gross, M. J., \& Brown, G. (2008). An empirical structural model of tourists and places: Progressing involvement and place attachment into tourism. Tourism Management, 29, 11411151.

Hidalgo, M. C., \& Hernandez, B. (2001). Place attachment: Conceptual and empirical questions. Journal of Environment Psychology, 21(3), 273-281.

Hing, B. S., Lee, E. J., Park, S. H., \& Yoo, S. H. (2010). The effects of department store loyalty programs on consumer relationship quality and relationship continuity intention. Journal of Korean Society of Clothing and Textiles, 34(10), 1621-1631.

Jung, J. H., \& Kim, Y. M. (2012). Influence of involvement in professional baseball \& sponsorship expectation on sponsor awareness, sponsor attitude \& product attitude. The Korean Journal of Physical Education, 51(6), 229-240.

Kandampulley, J., Mok, C., \& Sparks, B. (2001). Service quality management inhospitality. New York: The Howorth Hospitality Press.

Kang, D. W., \& Choi, C. G. (2012). Causality analysis of placeness conition and behavioral intention by visitors character. Journal of Korea Planners Association, 47(3), 363-379.

Kim, B., \& Jung J. H. (2018). Relationship between selfdetermination and relationship commitment, customer orientation, service quality, and relation continuity intention of fitness center customers. International Journal of Applied Sports Sciences, 29(1), 99-113.

Kim, B., \& Jung, J. H. (2017). Relationship between humor orientation and customer orientation, and the employees' trust, service quality, relationship quality, and relationship retention of employee in commercial sports center. International Journal of Applied Sports Sciences, 28(3), 636-651.

Kim, D. K. (2008). A study on the place attachment of residential environment. Ph.D. Dissertation, Seoul National University. 
Kim, D. Y., \& Park, H, S. (2015). The influence on the political efficacy from reading and writing of twitter users in terms of involvement. Korean Journal of Journalism \& Communication Studies, 59(1), 93-114.

Kim, H. J., \& Kwon, J. I. (2011). The effect of service benefits on trust, positive emotion and relation continuity intention for airline customer. Journal of Tourism and Leisure Research, 23(8), 285-304.

Kim, H. Y., \& Kim, S. J. (2014). A study on the effect of formative factors of placeness on place attachment, the value perception and visitor's behavioral intentions in museum. Journal of Arts Management and Policy, 30, 185-217.

Kim, H., Song, H. S., \& Kim, Y. E. (2015). Analysis of environmentally responsible behaviors based on a typology of activity involvement and place attachment: Focuses on visitors to Namhansanseong provincial park. Journal of the Korean Institute of Landscape Architecture, 43(3), 114-124.

Kim, K. R. (2016). The effects of TPB factor of participate in water Ski on place attachment and leisure continuance. Korean Society of Leisure \& Recreation, 40(4), 51-64.

Kim, M. J. (2017). Tourists perception of feeling of authentic, participation and place attachment through cultural heritages. The 48th Conference of Korea Academic Society of Tourism and Leisure, 317-323.

Kim, S. J. (2005). A study on the causal model of service quality, place attachment and destination loyalty in the cultural festival. Journal of the Economic Geographical Society of Korea, 8(2), 315-330.

Knez, I. (2005). Attachment and identity as related to a place and its perceived climate. Journal of Environment Psychology, 25, 207-218.

Kwon, Y. G., Kim, Y. J., Yoon, H. H. (2012). The influence of consumption value and service quality in resort on the consumer satisfaction. Korean Journal of Hotel Administration, 21(3), 69-85.

Kyle, G., Bricker, K., Graefe, A., \& Wickham, T. (2004a). An examination of recreationists' relationships with activities and settings. Leisure Sciences, 26(2), 123-142.

Kyle, G., Graefe, A., Manning, R., \& Bacon, J. (2004b). Effect of activity involvement and place attachment on recreationists' perceptions of setting density. Journal of Leisure Research, 36(2), 209-231.

Lee, B. H. (2012). Structural relationship among utilitarianhedonic shopping value, emotional responses, store attitude and loyalty of sporting goods consumer. Korean Journal of
Sports Science, 21(6), 915-930.

Lee, B. R., \& Hwang, K. J. (2017). Green restaurant consumers' place identity and ethical self-identity mediated by cognitive satisfaction affection loyalty and cooperation moderating effects of mindfulness. Journal of Consumer Studies, 28(3), 47-75.

Lee, J. H. (2011a). A study on the impact of users' place attachment on the value of public libraries. Journal of the Korean Society for Library and Information Science, 45(5), 209-234.

Lee, K. S., \& Jung, J. H. (2015). A study on the influence of innovative perception of $\&$ involvement in sports product on hedonic value, product attitude and purchase intention. Korean Journal of Sport Science, 26(2), 292-303.

Lee, T. (2011b). How recreation involvement, place attachment and conservation commitment affect environmentally responsible behavior. Journal of Sustainable Tourism, 19(7), 895-915.

Lim, B. J. (2014). The effect of mega event brand equity on place attachment: Focused on moderating effects of volunteer service performance. International Journal of Tourism and Hospitality Research, 28(12), 75-91.

Lyu, S. O., \& Oh, C. O. (2008). Assessing the causal linkages between recreation specialization and place attachment: A study of Texas Anglers. The Journal of Tourism Studies, 20(2), 3-23.

Manzo, L. C. (2005). For better or worse: Exploring multiple dimensions of place meaning. Journal of Environmental Psychology, 25(1), 67-86.

McAndrew. F. (1998). The measurement of 'rootdness' and the prediction of attachment to home-towns in college students. Journal of Environmental Psychology, 18(4), 409-417.

Ministry of Culture Sports and Tourism. (2017a). 2016 Sport white paper.

Ministry of Culture Sports and Tourism. (2017b). 2016 sport industry white paper.

Nunnally, J. C., \& Bernstein, I. H. (1994). Psychometric theory. NY: McGraw-Hill.

Pan, Y., \& Schimitt, B. (1996). Language and brand attitudes: Impact of script and sound matching in Chinese and English. Journal of Consumer Psychology, 5(3), 263-277.

Parasuraman, A., Zeithmal, V. A., \& Berry, L. L. (1988). SERVQUAL: A multiple item scale for measuring consumer perceptions of service quality. Journal of Retailing, 64(1), 12-40.

Park, J. B., \& Choi, J. W. (2018). A study on the motives of tour- 
ists, attachment and royalties in marine tourism: Focusing on the Boryeong-si Daecheon beach. Journal of Tourism Management Research, 22(3), 871-891.

Park, J. S., \& Jin, M. X. (2017). The economic value of stream edge space within a city depending on activity involvement and place attachment. Journal of Tourism Sciences, 41(7), 175-198.

Park, M. R., \& Kim, Y. I. (2014). A study of the brand equity of local food on place attachment as well as attitude of host region: Visitors to Abai village in Sokcho. Korean Journal of Tourism Research, 29(5), 99-115.

Peterson, G. L, Stynes, D. L., Rosenthal, D. H., \& Dwyer, J. F. (1985). Substitution in recreation choice behavior. In G. H. Stankey and S. F. McCool(Compilers). Proceedings Symposium on Recreation Choice Behavior. 19-30.

Prayag, G., \& Ryan, C. (2012). Antecedents of tourists' loyalty to Mauritius. Journal of Travel Research, 51(3), 342-356.

Proshansky, H. M., Fabian, A. K., \& Kaminof, R. (1983). Place identity: Physical world and socialization of the self. $J$. Environ. Psychol., 3, 57-83.

Sbpetim, S. (2012). Exploring the relationship among service quality, satisfaction, trust and store loyalty among retail customers. Journal of Competitiveness, 4(4), 16-35.

Schuett, M. (1993). Refining measures of adventure recreation involvement. Leisure Science, 15, 205-216.

Seok, K. H. (2010). The relationship between of the purchase conduct of service quality and lifestyles of the indoor swimming pool customers. The Korea Journal of Sports Science, 19(3), 813-825.

Son, B. M. (2016). The effects of the tourism image of Pyeongchang Winter Olympics on perceived value and community attachment: Focused on the moderating effect of in- volvement. International Journal of Tourism and Hospitality Research, 30(1), 163-183.

Son, E., \& Jun, J. K. (2014). The influence of place attachment on visit intention through the mediating of place brand attitude: Focused on Busan Yeongdo bridge. Journal of Tourism and Leisure Research, 26(9), 415-431.

Swann, W. B., De La Ronde, C., \& Hixon, J. G. (1994). Authenticity and positivity strivings in marriage and courtship. Journal of Personality and Social Psychology, 66, 857-869.

Swann, W. B., Stein-Seroussi, A., \& Giesler, R. B. (1992). Why people self-verify. Journal of Personality and Social Psychology, 62(3), 392-401.

Williams, D. R., \& Roggenbuck, J. W. (1989). Measuring place attachment: Some preliminary results. Leisure Research Symposium. Arlington, VA: National Recreation and Park Association

Williams, D. R., Roggenbuck, J. W., Patterson, M. E., \& Watson, A. E. (1992). Beyond the commodity metaphor: Examining emotional and symbolic attachment to place. Leisure Sciences, 14(1), 29-46.

Yoon, H. H. (2011). Influences of brand equity of rural tourism village on place attachment and pro-environmental behavior intentions. Ph.D. Dissertation, Kyonggi University.

Zaichkowsky, J. L. (1985). Measuring the involvement construct. Journal of Consumer Research, 12(3), 341-352.

Zeithaml, V. A., Berry, L. L., \& Parasuraman, A. (1996). The behavioral consequences of service quality. Journal of Marketing, 60(2), 31-46.

Zhang, J., Inbakaran, R. J., \& Jackson, M. S. (2006). Understanding community attitudes towards tourism and hostguest interaction in the urban-rural border region. Tourism Geographies, 8(2), 182-204. 


\section{사설실내수영장의 서비스품질과 관여도가 장소애착, 장소태도 및 관계지속의도에 미치는 영향 \\ 이광수(순천향대학교), 정정희(단국대학교)}

〔목적〕 이 연구의 목적은 실내수영장 고객이 지각하는 서비스품질과 관여도가 장소애착, 장소태도 및 관계 지속의도와 관계를 구조방정식모형을 통해 실증적으로 검증하는 것이다. 〔방법〕 이를 위해 서울에 소재한 실 내수영장 5 개소 남녀 회원 241 명을 대상으로 설문조사를 실시하였다. 표집방법은 편의표본추출법과 설문지 작성은 자기기입식으로 하였다. 제안한 구조모형을 검증하기 위해 IBM SPSSWIN Ver. 21.0과 AMOS 18.0 을 사용하였다.〔결과〕 첫째, 서비스품질은 장소정체성에 긍정적인 영향을 미쳤다. 둘째, 서비스품질은 장소 의존성에 긍정적인 영향을 미쳤다. 셋째, 관여도는 장소정체성에 긍정적인 영향을 미치지 않았다. 넷째, 관여 도는 장소의존성에 긍정적인 영향을 미치지 않았다. 다섯째, 장소정체성은 장소태도에 긍정적인 영향을 미쳤 다. 여섯째, 장소의존성은 장소태도에 긍정적인 영향을 미쳤다. 일곱째, 장소태도는 관계지속의도에 긍정적인 영향을 미쳤다.

주요어: 서비스품질, 관여도, 장소정체성, 장소의존성, 장소태도, 관계지속의도 